\title{
A COMPARISON OF METHODS FOR DETECTING THE EGGS OF ASCARIS, TRICHURIS, AND HOOKWORM IN INFANT STOOL, AND THE EPIDEMIOLOGY OF INFECTION IN ZANZIBARI INFANTS
}

\author{
DAVID GOODMAN,* HAMAD J. HAJI, QUENTIN D. BICKLE, REBECCA J. STOLTZFUS, JAMES M. TIELSCH, \\ MAHDI RAMSAN, LORENZO SAVIOLI, AND MARCO ALBONICO \\ Department of International Health, Johns Hopkins Bloomberg School of Public Health, Baltimore, Maryland; Pemba Public Health \\ Laboratory, Ivo de Carneri, Chake Chake, Pemba Island, Zanzibar, The United Republic of Tanzania; Department of Infectious \\ and Tropical Diseases,London School of Hygiene and Tropical Medicine, London, United Kingdom; Division of Nutritional \\ Sciences, Cornell University, Ithaca, New York; Division of Intestinal Parasites and Vector Control, World Health Organization,
} Geneva, Switzerland

\begin{abstract}
This study compared five methods for detecting the eggs of the human parasitic geohelminths Ascaris, Trichuris, and hookworm in infant stool, and describes the epidemiology of infection in infants from a parasite-endemic area. A total of 424 infants 5-11 months old were enrolled from three villages on Pemba Island, Zanzibar. Methods used included the Kato-Katz technique, formol ethyl acetate sedimentation, modified formol ethyl acetate sedimentation, modified Wisconsin floatation, and simple gravity sedimentation. Of methods used alone, Wisconsin floatation and simple gravity sedimentation each provided the highest sensitivity for detecting eggs of these three geohelminths $(89.6 \%)$. Of methods used in combination, the Kato-Katz technique/simple gravity sedimentation and Wisconsin floatation/simple gravity sedimentation each provided the highest sensitivity $(99.0 \%)$. Prevalence of geohelminth infection was $26.5 \%$. Between five and nine months of age the mean prevalence was $9.4 \%$, while at 10 and 11 months of age the mean prevalence was $43.4 \%$. Village prevalence varied from $3.6 \%$ to $43.8 \%$. Infant geohelminth infection can occur at a high prevalence, and what method is best depends on research specifics.
\end{abstract}

\section{INTRODUCTION}

Worldwide, there are approximately 3.5 billion infections with the parasitic geohelminths Ascaris, Trichuris, and hookworm. ${ }^{1}$ Most infections occur in developing countries, with the greatest morbidity in children and pregnant women. In school age children, geohelminth infection has been associated with poor nutritional status and iron-deficiency anemia, which causes concern that they are placed at increased risk of morbidity and mortality. ${ }^{2-6}$ For these reasons, coupled with the cost-effectiveness of anthelmintic delivery to children in this age group, research and interventions have been targeted toward school age children. ${ }^{7,8}$ In contrast, research and interventions targeted toward geohelminth infections in preschool age children have been limited.

The lack of focus on preschool age children is based on an assumed low prevalence and infection intensity, accompanied by a belief that low-intensity infections do not result in significant morbidity. ${ }^{9}$ However, the reasons to expand the focus to geohelminth infections in preschool age children are growing. Recently, mebendazole treatment of preschool age children was demonstrated to have a greater benefit on growth in children 6-30 months of age than in children 30-71 months of age, despite the younger children having lower egg counts. ${ }^{10}$ These data suggest that infections characterized by low egg counts can produce significant morbidity in young children.

Incomplete immune system development and a high metabolic demand make the youngest of these preschool age children, infants, a group that may be especially susceptible to growth effects induced by geohelminth infection. In addition, infants represent a unique population, largely having first

* Address correspondence to David Goodman, Florida Department of Health, 4052 Bald Cypress Way, Bin \#A13, Tallahassee, FL 32399. E-mail: dave_goodman@doh.state.fl.us time (incident) infections. Such incident infections may stimulate host responses qualitatively and/or quantitatively quite different from those observed in the repeated or chronic infections observed in older children. There is also increasing interest in immunomodulatory responses induced by helminthes. ${ }^{11}$ A particular focus of attention is on how such infections will influence the developing immune response of infants not only to childhood infections but also childhood vaccination.

Such interest in the potential clinical and immunologic importance and prevalence of geohelminth infection in infants explains our focus on a suitably sensitive method for helminth egg detection. The ease of its use in the field has established the Kato-Katz technique as the method of choice for the diagnosis of geohelminth infections when doing large field surveys. ${ }^{12}$ However, the usefulness of the Kato-Katz method in detecting infections in infants may be limited because the stools of breastfed infants tend to be more liquid and have relatively low egg counts.

In support of a research project to determine the clinical and immunologic impact of geohelminth infections in infants, a study was conducted to determine the best method, or combination of methods, to detect the eggs of geohelminth present in the stool of infants in a parsite-endemic area. Protocols were selected from those used both in human and veterinary medicine, and included the Kato-Katz method, as well as sedimentation and floatation methods. Some protocols were modified to facilitate applicability for the age group, use in the field, and screening large numbers. We report here the sensitivity of these methods and the prevalence of geohelminth infections observed in a community-based sample of more than 400 Zanzibari infants.

\section{MATERIALS AND METHODS}

Study population. This study was conducted on Pemba Island, Zanzibar, United Republic of Tanzania, at the Pemba 
Public Health Laboratory-Ivo de Carneri (PHL-IdC). Pemba has a length of 67 and a width of $22 \mathrm{~km}$. Long rains occur from mid March to May and short rains occur in November and December, with the dry seasons intervening. Temperature and humidity remain fairly stable throughout the year, with the average daytime temperature ranging from $22^{\circ} \mathrm{C}$ to $33^{\circ} \mathrm{C}$ and average relative humidity ranging from $61 \%$ to $89 \%$ (Goodman D, unpublished data). Fishing and farming are the major sources of economic activity on the island, and agriculture employs most of the work force.

Malaria transmission occurs throughout the island and is year round, peaking after the rainy seasons. Plasmodium falciparum is the predominant species. The helminths Schistosoma haematobium and Wuchereria bancrofti are endemic. Hookworms of the two genera Necator and Ancylostoma are present, with Necator the more prevalent of the two. ${ }^{13}$

Informed consent was obtained from the guardians of all enrolled infants. Ethical review committees of Johns Hopkins Bloomberg School of Public Health and Hygiene, Cornell University, London School of Hygiene and Tropical Medicine, and the Ministry of Health of Zanzibar reviewed and approved this research.

Stool collection. Four hundred twenty-four infants 5-11 months of age were enrolled during one of the intervening dry seasons between the months of August and October 2002. Infants were recruited from three villages (Wesha, Pujini, and Vitongoji) located north to south in the middle of the island. Age was determined by maternal report, and confirmed by birth cards when available. Those children less than five months of age and more than 12 months of age were excluded from participation, as were children with an undeterminable age. No other exclusion criteria were used.

Stool samples were collected from each enrolled child on two days in the following manner. On the initial home visit, the infant's guardian was provided with a plastic container to be collected the following day. When a day 1 container with a stool sample was retrieved, a day 2 container was then provided for collection on the following day. The day 1 stool was brought back to the public health laboratory where it was stored at $4^{\circ} \mathrm{C}$. Once the day 2 stool was collected and brought to the public health laboratory, the matching day 1 sample was removed from the refrigerator, and the samples were combined and weighed. Three days was the longest amount of time that a day 1 stool was kept in the refrigerator before analysis, which was approximately half the length of time before deterioration of hookworm eggs was observed (Goodman D, unpublished data).

Diarrhetic stool. Prior to processing, each stool was examined and subjectively determined whether it was diarrhetic. Diarrhetic stools were included for analysis because it was expected that their exclusion would have resulted in the loss of a significant number of children. To allow the use of diarrhetic stools with the Kato-Katz method, diarrhetic stools were transferred directly to the a template using a disposable polypropylene transfer pipette. For all other methods, diarrhetic stool was weighed similar to non-diarrhetic stool.

Stool examination. Ten grams was determined to be the minimum amount of stool required to perform the five methods selected for comparison. If the combined day 1 and day 2 stool mass was less than 10 grams, or if only one day of stool was collected, the sample was examined by the Kato-Katz method alone, and was excluded from this analysis. Those stools with a combined mass $\geq 10$ grams were divided into required masses for each of the five compared methods.

The methods selected for comparison were the modified Kato-Katz thick smear, a method primarily used in human medicine; two versions of the formol ethyl acetate sedimentation, a method commonly used in both human and veterinary medicine; a modified Wisconsin floatation, a method primarily used in veterinary medicine; and a simple gravity sedimentation, a method not commonly used in either human or veterinary medicine. All egg counts were recorded as total counts for each of the three geohelminths and then converted to eggs per gram (epg) of stool as appropriate. Mebendazole (Keko Pharmaceutical Industries, Dar es Salaam, Tanzania) was provided to all infants with a geohelminth infection at a dosage of $100 \mathrm{mg}$, twice a day, for three days.

Kato-Katz method. Duplicate Kato-Katz slides were made from the combined day 1 and the day 2 stools. The preparation of each slide was as per the World Health Organization (WHO) protocol. ${ }^{12}$ One gram of stool was used for the two slides. Number 120 -sized nylon mesh screen was used for filtering the stool, and a 41.7-mg plastic template was used.

Formol ethyl acetate method. The protocol developed by Ritchie $^{14}$ and Young and others ${ }^{15}$ and described by Ash and Orihel $^{16}$ was followed using $1 \mathrm{~g}$ of stool; all of the sediment was examined. A tea strainer was initially used as a screening stool, but it allowed a large amount of sediment to pass through, which hindered accurate reading of slides. Squares of the screen used for the Kato-Katz method were used in place of the tea strainer, folded into quarters, and opened to form a funnel. This resulted in a decrease in sediment and an increase in the ability to read the slide. No conversion was needed to obtain an estimated epg.

Modified formol ethyl acetate method. The modified protocol is based on the work of Allen and Ridley, ${ }^{17}$ as described by Cheesbrough, ${ }^{18}$ using one gram $\mathrm{g}$ of stool. ${ }^{17,18}$ This method differed from the formaol ethyl acetate method: the water wash/centrifugation step prior to the addition of formalin and ethyl acetate was eliminated and the centrifugation speed was increased from $242 \times g$ to $1,058 \times g$. As with the above protocol, use of a mesh screen instead of a tea strainer reduced sediment and improved readability. No conversion was needed to obtain an estimated epg.

Modified Wisconsin method. The protocol used was based on that described by Cox and Todd ${ }^{19}$ and Egwang and Slocombe. ${ }^{20}$ Briefly, five grams of stool was weighed and placed in a polypropylene cup. Water was then added to give a total volume of $30 \mathrm{~mL}$, and a tongue depressor was used to macerate the stool and create a fecal slurry. The slurry was then passed through a tea strainer. While hand-swirling the slurry, $12 \mathrm{~mL}$ of the filtered slurry was transferred into a $12-\mathrm{mL}$ centrifuge tube. The samples were then centrifuged at $256 \times$ $g$ for 3 minutes. The supernatant was removed, with care being taken not to lose the fine sediment of the pelleted material. The tube was then filled approximately half way with Sheathers sugar solution (specific gravity $=1.27$ ), and the pellet was dispersed using a wooden applicator stick. The tube was then topped off with Sheathers sugar solution until a meniscus was formed, and a $22 \times 22 \mathrm{~mm}$ coverslip was placed on top. The samples were then centrifuged at $256 \times g$ for 5 minutes. The coverslips were transferred to slides and 
the entire coverslip was examined. To obtain an estimated epg, the total egg count was divided by 2 .

Simple gravity sedimentation method. The simple gravity sedimentation protocol was initially developed using stool from healthy infants from the United States spiked with eggs of the ovine trichostrongyle Haemonchus contortus and eggs of the swine trichuroid Trichuris suis. The developed protocol was as follows: 2 grams of stool were weighed and placed in a 100-mL polypropylene cup. The stool was then macerated in approximately $50 \mathrm{~mL}$ of water to create a fecal slurry. The slurry was passed through a tea strainer into a polycarbonate champagne flute (Tyco, Eagan, MN). A wooden tongue depressor was used to compress the slurry remaining in the strainer to ensure all excess water was passed through the strainer into the flute. Approximately $50 \mathrm{~mL}$ of water was added to the cup that held the slurry, which was then passed through the strainer into the flute. Water was added to the flute until it was full, and it was then left to sit for 30 minutes. The supernatant was removed from the sedimented material. The flute was then topped with water and left undisturbed for 30 minutes. The supernatant was removed from the sediment, and the sediment was transferred to a petri dish $(100 \times 15 \mathrm{~mm}$, Falcon Optilux ${ }^{\mathrm{TM}}$; Becton Dickinson, Franklin Lakes, NJ). Water was then added to the flute, swirled, and transferred to the petri dish. This process was repeated until all sediment was transferred to the petri dish. The dish was then placed on the stage of an inverted microscope (TMS with a mechanical stage; Nikon, Tokyo, Japan) for examination. To assist with reading, a frame to fit within the mechanical stage and around the petri dish was cut from cardboard. The sediment in the dish was allowed to settle for 2-3 minutes before reading. To obtain an estimated epg, total egg counts were divided by 2 .

Statistical analysis. Only those children who provided two days of stool samples, with a combined day 1 and day 2 stool mass of at least 10 grams, were used in analysis. Stata version 8.0 (Stata Corporation, College Station, TX) was used for statistical analysis. A one-sample test for differences in proportions was used to determine whether the prevalence found by each method and combinations of methods were statistically significantly different from the prevalence calculated by
Kato-Katz method alone. McNemar's chi-square test was used to determine if agreement was significantly different between the duplicate Kato-Katz protocol and each method or combination of methods. The sensitivity of each method and combinations of methods were calculated based on comparison with those results obtained by all methods combined. Combinations of methods were limited to two based on what would be practical to apply in the laboratory. Multiple logistic regression was used to describe the relationship of diarrhetic stool with geohelminth infection. Statistical significance for all analyses was determined at the 5\% alpha level.

\section{RESULTS}

The enrolled 424 infants were composed of 231 girls (54\%), and 193 boys ( $46 \%$ ), with 147 infants between 5 and 8 months of age (35\%), and 277 infants between 9 and 11 months of age $(65 \%)$. The data from 354 infants, whose composition was almost identical to that observed for the full cohort, were used for analysis.

Mass of stool. Four hundred eight $(96.2 \%)$ of 424 enrolled infants provided a day 1 stool and $391(92.2 \%)$ provided 2 days of stool. Of those infants who provided 2 days of stool, $354(90.5 \%)$ had a combined stool mass $\geq 10$ grams. The median (range) of the mass of stool from one day and two days of collection were 12.3 grams $(0.2-45.7)$ and 25.1 grams (2.3-88.5), respectively.

Prevalence. Prevalence of geohelminth infection, as determined by each method or combination of methods, is shown in Table 1 . When methods used alone were compared, the duplicate Kato-Katz, Wisconsin, and sedimentation protocols provided a similar prevalence for infection with any of the three worms, which was significantly greater than that observed for the two formol ethyl acetate protocols. Sedimentation provided the highest prevalence of Ascaris (12.1\%) of methods used alone, although it was lower than the prevalence observed from all methods combined. The Kato-Katz protocol showed the highest prevalence of Trichuris, which was significantly higher than any other single method, but still lower than that observed from all methods combined. Sedi-

TABLE 1

Prevalence (95\% confidence interval) of geohelminth infection by method*

\begin{tabular}{|c|c|c|c|c|}
\hline Method & Any one or more geohelminths & Ascaris & Trichuris & Hookworm \\
\hline KK & $23.4(19.0-27.8)$ & $10.2(7.1-13.3)$ & $22.6(18.3-26.9)$ & $6.1(3.6-8.5)$ \\
\hline $\mathrm{W}$ & $23.7(19.3-28.1)$ & $9.1(6.1-12.1)$ & $16.8(12.9-20.7)+\neq$ & $6.3(3.8-8.9)$ \\
\hline $\mathrm{S}$ & $23.7(19.3-28.1)$ & $12.1(8.8-15.5)$ & $14.3(10.7-18.0) \dagger+$ & $6.9(4.3-9.5)$ \\
\hline $\mathrm{F}$ & $18.7(14.7-22.8) \dagger+$ & $8.3(5.4-11.1)$ & $12.1(8.8-15.5) \dagger+$ & $5.8(3.4-8.2)$ \\
\hline $\mathrm{MF}$ & $19.8(15.7-24.0) \dagger+$ & $9.1(6.1-12.1)$ & $14.6(11.0-18.3) \dagger+$ & $5.0(2.7-7.2)$ \\
\hline $\mathrm{KK}+\mathrm{W}$ & $24.0(19.6-28.4)$ & $10.5(7.3-13.6)$ & $22.9(18.5-27.2)$ & $6.3(3.8-8.9)$ \\
\hline $\mathrm{KK}+\mathrm{S}$ & $26.2(21.6-30.7) \div$ & $13.2(9.7-16.7) \ddagger$ & $23.1(18.8-27.5)$ & $7.6(4.8-10.4) \div$ \\
\hline $\mathrm{KK}+\mathrm{F}$ & $25.1(20.6-29.6) \ddagger$ & $11.0(7.8-14.3)$ & 23.4 (19.0-27.8) & $6.6(4.0-9.2)$ \\
\hline $\mathrm{KK}+\mathrm{MF}$ & $24.0(19.6-28.4)$ & $10.7(7.5-13.9)$ & $22.9(18.5-27.2)$ & $6.3(3.8-8.9)$ \\
\hline $\mathrm{W}+\mathrm{S}$ & $26.2(21.6-30.7) \div$ & $12.7(9.2-16.1) \ddagger$ & $17.4(13.4-21.3) \dagger+$ & $7.6(4.8-10.4) \div$ \\
\hline $\mathrm{W}+\mathrm{F}$ & $24.8(20.3-29.3)$ & $10.2(7.1-13.3)$ & $17.4(13.4-21.3) \dagger+$ & $6.6(4.4-9.2)$ \\
\hline $\mathrm{W}+\mathrm{MF}$ & $23.7(19.3-28.1)$ & $9.9(6.8-13.0)$ & $16.8(12.9-20.7)+\neq$ & $6.3(3.8-8.9)$ \\
\hline $\mathrm{S}+\mathrm{F}$ & $25.1(20.6-29.6)$ & $12.1(8.8-15.5)$ & 15.7 (11.9-19.5)十斗 & $7.4(4.6-10.1) \ddagger$ \\
\hline $\mathrm{S}+\mathrm{MF}$ & $25.3(20.8-29.8)$ & $12.4(9.0-15.8) \ddagger$ & $16.8(12.9-20.7) \dagger+$ & $7.2(4.5-9.8)$ \\
\hline $\mathrm{F}+\mathrm{MF}$ & $22.9(18.5-27.2)$ & $10.2(7.1-13.3)$ & $15.4(11.7-19.2)+\neq$ & $6.3(3.8-8.9)$ \\
\hline All & $27.3(22.6-31.9)$ & $13.6(10.0-17.2)$ & $24.0(19.5-28.5)$ & $7.6(4.8-10.4)$ \\
\hline
\end{tabular}

$* \mathrm{KK}=$ Kato-Katz; $\mathrm{W}=$ modified Wisconsin; $\mathrm{S}=$ sedimentation; $\mathrm{F}=$ formol ethyl acetate; $\mathrm{MF}=$ modified formol ethyl acetate; All = all five methods combined. Numbers in bold represen the highest prevalence recorded for that column.

$\dagger$ Statistically significantly $(P \leq 0.05)$ different from prevalence using duplicate Kato-Katz slides.

$\doteqdot$ Agreement between results of the Kato-Katz and method(s) significantly $(P \leq 0.05)$ different. 
mentation was the single method that provided the highest prevalence for hookworm, although like other methods used alone, it showed a prevalence lower than that observed for all methods combined.

Combinations of methods tended to perform better than any one method used alone. The combinations of the KatoKatz and sedimentation methods and the Wisconsin and sedimentation methods showed the highest prevalence of infection with any geohelminth. However, these combinations still showed a prevalence that was less than that found by combining the results of all methods.

The Kato-Katz/sedimentation combination provided the highest observed prevalence for infection with Ascaris, and matched the prevalence found by all methods. This combination also resulted in prevalence estimates significantly greater than that observed by the Kato-Katz protocol alone. The combination of the Kato-Katz and formol ethyl acetate methods showed the highest prevalence of Trichuris, which was equivalent to the prevalence found by all methods. However, this combination did not result in a prevalence that was significantly greater than the duplicate Kato-Katz method alone. The combinations of sedimentation/Kato-Katz, sedimentation/Wisconsin, and sedimentation/formol ethyl acetate methods provided the highest observed prevalence for hookworm, which was equivalent to the prevalence found by all methods. These combinations provided a prevalence that was significantly greater than that observed by the Kato-Katz method alone.

Prevalence by age. The prevalence of geohelminth infection by age is shown in Figure 1. The prevalence of Ascaris and Trichuris were similar between 5 and 9 months of age (approximately $6 \%$ ), with a mean prevalence of $9.4 \%$ (95\% confidence interval $[\mathrm{CI}]=5.1-13.7 \%$ ) for infection with any one or more of the geohelminths. At 10 and 11 months of age, prevalence sharply increased to a mean prevalence of $43.4 \%$ $(95 \% \mathrm{CI}=36.1-50.7 \%)$ for infection with any of the geohelminth. In children more than nine months of age Trichuris becomes strikingly more prevalent than infection with any other worm. Average egg counts (epg) follow a similar age pattern. Hookworm eggs were not detected in the stool until infants were eight months of age, and remained below a prevalence of $15 \%$ at all ages.

Prevalence by village. There was a considerable variability in prevalence of any one or more geohelminth infections between the three study villages, with prevalences of $43.8 \%$ $(95 \% \mathrm{CI}=35.4-52.2 \%)$ in Wesha, $17.7 \%$ (95\% CI $=12.3-$ $23 \%)$ in Vitongoji, and 3.6\% (95\% CI $=0-10.9 \%)$ in Pujini.

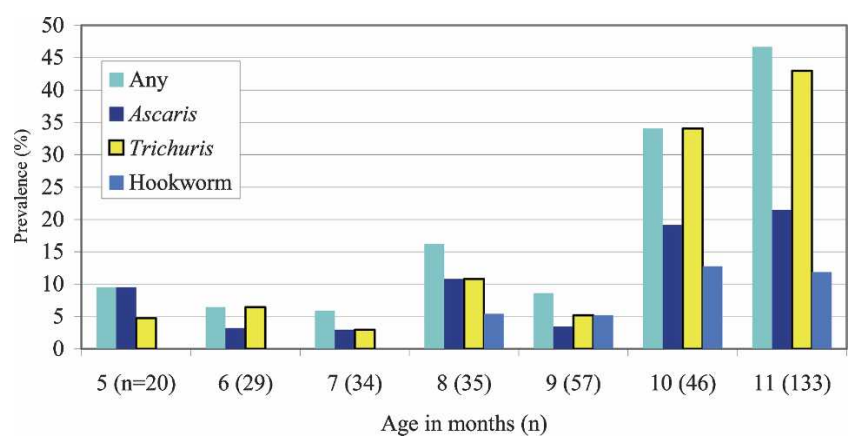

FIGURE 1. Prevalence of geohelminth infection by age. This figure appears in color at www.ajtmh.org.
Prevalence of co-infection. The distribution of co-infections among infected infants is shown in Figure 2. Ascaris and Trichuris was the most common co-infections observed in infants. Ascaris and hookworm were never observed to occur together without Trichuris infection also present.

Egg counts. Eggs per gram of stool from the averaged two Kato-Katz slides are shown in Table 2. In general, the epg from the Kato-Katz method tended to be higher (2-14 times higher) than those observed using other methods. This is consistent with previous observations comparing the Kato-Katz method with the formol ether sedimentation method. ${ }^{21}$ Of the 48 children with Ascaris infection, 29 passed unfertilized eggs, of whom 24 passed unfertilized eggs only and 5 passed a mixture of fertilized and unfertilized eggs.

Sensitivity. A gold standard does not exist for the detection of geohelminth infection. Thus, a combination of all five methods evaluated here was used as a gold standard to estimate the sensitivity of each method and combination of methods. The calculated sensitivities for the compared methods are shown in Table 3.

Diarrhetic stool. Diarrhetic stool was observed in $16.0 \%$ of the samples, for at least one of the two days of collection. Only $3.1 \%$ of the infants had diarrhetic stool for both of their submitted samples. A child having diarrhetic stool on either or both of the collection days was associated with a decreased risk (odds ratio $[\mathrm{OR}]=0.5,95 \% \mathrm{CI}=0.2-1.2$ ) of observing eggs in stool examinations for any of the worms, but this association disappeared once adjusted for age, a confounder in this analysis (Table 4). The protective effect of diarrhea for the observation of Trichuris eggs remained (OR $=0.4,95 \%$ $\mathrm{CI}=0.2-1.2, P=0.09)$, although the statistical evidence of a true association was weak.

\section{DISCUSSION}

The mass of stool that an infant produces determines the method or methods that can be used for determining geohelminth infection status. For example, use of 10 grams of stool for the Wisconsin method may have improved the sensitivity of this method, but this would have also meant the exclusion of a large number of children (approximately 16\%). The sum of the observed day 1 and day 2 masses of stool may be misleading because the median two-day total was approximately twice that observed for the one-day mass. On an individual basis, however, the mass collected from one day was not associated with the mass of stool collected on the second day. The ideal method or combination of methods should be capable of providing a high sensitivity and throughput, while requiring only a small amount of stool and minimizing the exclusion of children.
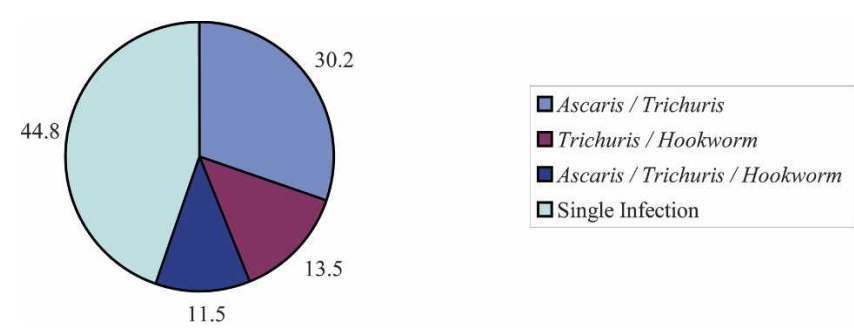

FiguRE 2. Prevalence (\%) of single geohelminth infections and geohelminth coinfections among 96 infected infants. This figure appears in color at www.ajtmh.org. 
TABLE 2

Eggs per gram of stool for each of the geohelminth infections in infants, as determined by the average of the egg counts from the duplicate Kato-Katz slides

\begin{tabular}{lccccr}
\hline Geohelminth & Median & Range & Bottom 25\% & Middle 50\% & Top 25\% \\
\hline Ascaris & 420 & $12-13,272$ & $<120$ & $120-1,260$ & $>1,260$ \\
Trichuris & 180 & $12-2,520$ & $<60$ & $60-520$ & $>520$ \\
Hookworm & 204 & $12-1,644$ & $<96$ & $96-360$ & $>360$ \\
\hline
\end{tabular}

The duplicate Kato-Katz method with stool samples from two days provided the highest sensitivity for Trichuris infection when methods were considered alone. The initial motivation for using two days of stool was to address the sporadic egg shedding of Trichuris spp. This decision was supported by the approximate $30 \%$ increase in sensitivity by making KatoKatz slides on two days of stool over a single Kato Katz slide on a single day of stool (Goodman D, Tielsch JM, Stoltzfus $\mathrm{RJ}$, unpublished data). This increased sensitivity is likely the result of overcoming the combined effect of sporadic egg shedding and uneven distribution of eggs in the stool. In addition, the infant stools were often fibrous, which can hinder the performance of the Kato-Katz method. It is possible that making slides on two days of stool helped to overcome this limitation.

The sedimentation method provided the highest sensitivity for hookworm and Ascaris when methods were considered alone. The reasons for its poor performance in detecting Trichuris eggs are not clear. It is possible the eggs themselves floated, or preferentially adhered to stool particulate that floated, resulting in eggs being lost in decanting. The finalized method is similar to that of Hoffman and others ${ }^{22}$ and nearly identical to that of Faust and Meleney, ${ }^{23}$ although these references were not found until after the development and implementation of the protocol used in this study. Although the sedimentation method shows superior sensitivity, it has same problem reported by Hoffman and others; it is timeconsuming when compared with other methods and requires adequate space to house the sedimentation flutes.

TABLE 3

Sensitivity (\%) of each method or combination of methods when the results of all methods combined is considered the gold standard*

\begin{tabular}{lcccc}
\hline \multicolumn{1}{c}{ Method } & $\begin{array}{c}\text { Any } \\
\text { geohelminth }\end{array}$ & Ascaris & Trichuris & Hookworm \\
\hline KK & 88.5 & 77.1 & $\mathbf{9 6 . 5}$ & 81.5 \\
W & $\mathbf{8 9 . 6}$ & 68.8 & 71.8 & 85.2 \\
S & $\mathbf{8 9 . 6}$ & $\mathbf{9 1 . 7}$ & 61.2 & $\mathbf{9 2 . 6}$ \\
F & 70.8 & 62.5 & 51.8 & 77.8 \\
MF & 75.0 & 68.8 & 62.4 & 66.7 \\
KK + W & 90.6 & 79.2 & 97.7 & 85.2 \\
KK + S & $\mathbf{9 9 . 0}$ & $\mathbf{1 0 0}$ & 98.8 & $\mathbf{1 0 0}$ \\
KK + F & 94.8 & 83.3 & $\mathbf{1 0 0}$ & 88.9 \\
KK + MF & 90.6 & 81.3 & 97.7 & 85.2 \\
W + S & $\mathbf{9 9 . 0}$ & 95.8 & 74.1 & $\mathbf{1 0 0}$ \\
W + F & 93.8 & 77.1 & 74.1 & 88.9 \\
W + MF & 89.6 & 75.0 & 71.8 & 85.2 \\
S + F & 94.8 & 91.7 & 67.1 & $\mathbf{1 0 0}$ \\
S + MF & 95.8 & 93.8 & 71.8 & 96.3 \\
F + MF & 86.5 & 77.1 & 65.9 & 85.2 \\
\hline
\end{tabular}

$* \mathrm{KK}=$ duplicate Kato Katz; $\mathrm{W}=$ modified Wisconsin; $\mathrm{S}=$ sedimentation; $\mathrm{F}$ = formo ethyl acetate; $\mathrm{MF}=$ modified formol ethyl acetate. Numbers in bold represent the highest sensitivity of the methods for each geohelminth or any one or more geohelminth. Single methods are considered separately from combinations of methods.
TABLE 4

Odds ratio of infection in children with diarrhetic stool compared with normal stool, adjusted for age*

\begin{tabular}{lclc}
\hline Geohelminth & Adjusted odds ratio & \multicolumn{1}{c}{$95 \% \mathrm{CI}$} & $P$ \\
\hline Any & 0.51 & $0.21-1.24$ & 0.14 \\
Ascaris & 0.83 & $0.30-2.3$ & 0.72 \\
Trichuris & 0.41 & $0.15-1.2$ & 0.09 \\
Hookworm & 0.28 & $0.04-2.2$ & 0.22 \\
\hline * CI = confidence interval. & & &
\end{tabular}

The prevalence of $43.8 \%$ observed in one of the study villages surpasses what is commonly observed in school age children from other parasite-endemic areas. The impact of exposure to these infections during infancy may be significant. This is a time when the immune system is still incomplete in its development, and the infant can ill afford diversions of resources from their growth and development.

The sharp increase in prevalence that occurs once children reach 10 months of age suggests modified risk factors. Possible factors are increased individual motility and weaning from breast milk to intake of solids, which carry an increased risk of ingestion of infective stages.

The large variation in prevalence observed between the three villages occurred within a short time frame and appears to be seasonally independent. Pockets of cultural or socioeconomic and environmental differences may exist between the areas, even though geographically they are close to one another. It is also possible that the age structure of the villages was responsible for the observed differences in prevalence; the village with the highest prevalence and the village with the intermediate prevalence were very similar in their age structure (median age $=10$ months), but the village with the lowest prevalence had a markedly younger age structure (median age $=8$ months) than the other two villages. It should be noted that the differences in median ages between the lowest prevalence village and the other two villages span the observed sharp age-specific increase in prevalence.

Co-infections represented approximately $55 \%$ of infections, with Ascaris/Trichuris being the most common combination. Of note is that hookworm infection never occurred without the presence of another infection. Coinfections are also common in school age children, but the increasing infection with hookworm contributes to a triple infection becoming the most prevalent co-infection in this age group on Pemba Island. ${ }^{24}$

Based on WHO guidelines, approximately $25 \%$ of infants with Ascaris and Trichuris infections and approximately $10 \%$ of infants with hookworm infections fall into the moderate egg count range. ${ }^{25}$ These guidelines for classification are based on clinical disease in school age children, and need to be re-examined for infections in infants, whose response to geohelminth infection is likely quite different than those in school age children. Infants also represent a group that is composed of a large number of incident infections that may impact the type of response mounted and associated clinical outcomes. ${ }^{26,27}$

The ability to identify unfertilized Ascaris eggs accounted for a large part of the superiority of the sedimentation method in identifying this infection. The Kato-Katz protocol identified 12 of 13 Ascaris infections identified by the sedimentation method when the ova where fertilized; but identi- 
fied only 15 of 24 Ascaris infections identified by the sedimentation method when the ova where unfertilized.

Since 53 (approximately 15\%) of the stools received were diarrhetic, we adopted methods suitable for use with such stools because they represent a significant proportion of the stools and also because children with diarrhetic stools may represent a clinically and immunologically important group. An apparent protective association between diarrhetic stool and geohelminth infection may be due to inherent difficulty in isolating eggs from diarrhetic stool, reduced actual fecal mass examined, or increased diarrhetic fecal output. The strongest protective effect was observed in relation to Trichuris infection, for which the Kato-Katz protocol was most sensitive. This is the most likely affected by diarrhetic stool consistency. However, the prevalence of Trichuris infection, as determined by the Kato-Katz method, in those infants with diarrhetic stool on one day and not the other is similar to that of infants with diarrhetic stool on both days (11\%). It should not be discounted that the responses that lead to diarrhetic stool may also play a role in limiting egg production or worm expulsion. In animal models, expulsion of intestinal parasitic nematodes is associated with responses that increase gut motility and intestinal muscular contractions. ${ }^{28,29}$ These same processes may result in diarrhetic stool.

Diarrhetic stool did not affect the sedimentation method, which although it has a lower sensitivity for Trichuris overall, was able to identify all infections in diarrhetic stool samples. The duplicate Kato-Katz slides for two days of stool showed good results with diarrhetic stools, but not as good as the sedimentation method. The Wisconsin method showed poor results with diarrhetic stools, perhaps because the specific gravity was affected by the increased water volume in the stool.

Which method is best to use will depend on researchspecific issues, including the research question, the sensitivity required, the expected egg counts, and geohelminth species of interest. In an area where Trichuris is the dominant species or is the sole species of interest, Kato-Katz slides for two days of stool collection may have sufficiently high sensitivity. If the research focus is on hookworm or Ascaris, the sedimentation method may be most suitable. If interest is in identifying true negative samples, or in identifying all infections, a combination of methods is likely required.

Little is understood about the transmission and risk factors for infection in infants. This work demonstrates that geohelminth infections in infants can occur at significant prevalences, and supports a coherent treatment strategy for children less than two years of age. In addition, the age-specific increase in the prevalence of geohelminth infections suggests a potential intervention point. Additional research to explore how biologic, environmental, and developmental changes surrounding the infant could provide an answer on how to delay infection in parasite-endemic areas beyond this time of vulnerability.

Received January 27, 2005. Accepted for publication July 19, 2005.

Acknowledgments: We thank Dr. Clive Shiff for his help in developing the simple gravity sedimentation, as well as the excellent field and lab staff of the PHL-IdC, and the community of Pemba Island.

Financial support: This study was supported by a grant from the Wellcome Trust.
Authors' addresses: David Goodman and James M. Tielsch, Department of International Health, Johns Hopkins Bloomberg School of Public Health, 615 North Wolfe Street, Baltimore, MD 21205. Hamad J. Haji and Mahdi Ramsan, Pemba Public Health Laboratory, Ivo de Carneri, Box 122, Chake Chake, Pemba Island, Zanzibar, The United Republic of Tanzania. Quentin D. Bickle, Department of Infectious and Tropical Diseases London School of Hygiene and Tropical Medicine, Keppel Street, London, United Kingdom WC1E 7HT. Rebecca J. Stoltzfus, Division of Nutritional Sciences Cornell University, Savage Hall, Ithaca, NY 14853. Lorenzo Savioli and Marco Albonico, Division of Intestinal Parasites and Vector Control, World Health Organization, Geneva, Switzerland.

\section{REFERENCES}

1. Chan MS, 1997. The global burden of intestinal nematode infections-Fifty years on. Parasitol Today 13: 438-443.

2. Hadju V, Abadi K, Stephenson LS, Noor NN, Mohammed HO, Bowman DD, 1995. Intestinal helminthiasis, nutritional status, and their relationship; a cross-sectional study in urban slum school children in Indonesia. Southeast Asian J Trop Med Public Health 26: 719-729.

3. Stoltzfus RJ, Albonico M, Tielsch JM, Chwaya HM, Savioli L, 1997. School-based deworming program yields small improvement in growth of Zanzibari school children after one year. $J$ Nutr 127: 2187-2193.

4. Saldiva SR, Silveira AS, Philippi ST, Torres DM, Mangini AC, Dias RM, da Silva RM, Buratini MN, Massad E, 1999. AscarisTrichuris association and malnutrition in Brazilian children. Paediatr Perinat Epidemiol 13: 89-98.

5. Stoltzfus RJ, Chwaya HM, Tielsch JM, Schulze KJ, Albonico M, Savioli L, 1997. Epidemiology of iron deficiency anemia in Zanzibari schoolchildren: the importance of hookworms. Am J Clin Nutr 65: 153-159.

6. Persson V, Ahmed F, Gebre-Medhin M, Greiner T, 2000. Relationships between vitamin $\mathrm{A}$, iron status and helminthiasis in Bangladeshi school children. Public Health Nutr 3: 83-89.

7. The Partnership for Child Development, 1998. Cost of schoolbased drug treatment in Tanzania. Health Policy Plan 13: 384396.

8. Anderson RM, 1980. The dynamics and control of direct life cycle helminth parasites. Biomath 39: 278-322.

9. Crompton DWT, Nesheim MC, 2002. Nutritional impact of intestinal helminthiasis during the human life cycle. Annu Rev Nutr 22: 35-59.

10. Stoltzfus RJ, Chway HM, Montresor A, Tielsch JM, Jape JK, Albonico M, Savioli L, 2004. Low dose daily iron supplementation improves iron status and appetite but not anemia, whereas quarterly anthelminthic treatment improves growth, appetite and anemia in Zanzibari preschool children. $J$ Nutr 134: 348-356.

11. Maizels RM, Yazdanbakhsh M, 2003. Immune regulation by helminth parasites: cellular and molecular mechanisms. Nat Rev Immunol 3: 733-744.

12. World Health Organization, 1991. Basic Laboratory Methods in Medical Parasitology. Geneva: World Health Organization.

13. Albonico M, Stoltzfus RJ, Savioli L, Tielsch JM, Chwaya HM, Ercole E, Cancrini G, 1998. Epidemiological evidence for a differential effect of hookworm species, Ancylostoma duodenale or Necator americanus, on iron status of children. Int $J$ Epidemiol 27: 530-537.

14. Ritchie LS, 1948. An ether sedimentation technique for routine stool examinations. Bull US Army Med Department 8: 326.

15. Young KH, Bullock SL, Melvin DM, Spruill CL, 1979. Ethyl actetate as a substitute for diethyl ether in the formalin-ether sedimentation technique. J Clin Microbiol 10: 852-853.

16. Ash LR, Orihel TC, 1991. Parasites: A Guide to Laboratory Procedures and Identification. Chicago: American Society of Clinical Pathologists.

17. Allen AHV, Ridley DS, 1970. Further observations on the formol-ether concentration-technique for faecal parasites. J Clin Pathol 23: 545-546.

18. Cheesbrough M, 1999. District Laboratory Practices in Tropical 
Countries. Part I. Tropical Health Technology. Cambridge, United Kingdom: Cambridge University Press.

19. Cox DD, Todd AC, 1962. Survey of gastrointestinal parasitism in Wisconsin dairy cattle. J Am Vet Med Assoc 141: 706-709.

20. Egwang TG, Slocomb JO, 1982. Evaluation of the CornellWisconsin centrifugal flotation technique for recovering trichostrongylid eggs from bovine feces. Canad J Comp Med 46: 133-137.

21. Ebrahim A, El-Morshedy H, Omer E, El-Daly S, Barakat R, 1997. Evaluation of the Kato Katz thick smear and formol ether sedimentation techniques for quantitative diagnosis of Schistosoma mansoni infection. Am J Trop Med Hyg 57: 706-708.

22. Hoffman WA, Pons JA, Janer JL, 1934. The sedimentation concentration method in schistosomiasis mansoni. $P R J$ Public Health Trop Med 9: 283-291.

23. Faust E, Meleney H, 1924. Studies on Schistosomiasis japonica. Am J Hyg 3: 218-219.

24. Booth M, Bundy DAP, Albonico M, Chwaya HM, Alawi KS, Savioli L, 1998. Associations among multiple geohelminth species infections in schoolchildren from Pemba Island. Parasitology 116: 85-93.
25. World Health Organization, 1998. Guidelines for the Evaluation of Soil-Rransmitted Helminthiases and Schistosomiasis at Community Level. Geneva: World Health Organization. WHO document WHO/CTD/SIP/98.1.

26. Bancroft AJ, Else KJ, Grencis RK, 1994. Low-level infection with Trichuris muris significantly affects the polarization of the CD4 response. Eur J Immunol 24: 3113-3118.

27. Roepstorff A, Eriksen L, Slotved HC, Nansen P, 1997. Experimental Ascaris suum infections in the pig: worm population kinetics following single inoculations with 3 doses of infective eggs. Parasitology 115: 443-452.

28. Vallence BA, Blennerhassett PA, Deng Y, Matthaei KI, Young IG, Collins SM, 1999. IL-5 contributes to worm expulsion and muscle hypercontractility in a primary $T$. spiralis infection. Am J Physiol 277: G400-G408.

29. Khan WI, Richard M, Akiho H, Blennerhasset PA, Humphreys NE, Grencis RK, van Snick J, Collins SM, 2003. Modulation of intestinal muscle contraction by interleukin-9 (IL-9) or IL-9 neutralization: correlation with worm expulsion in murine nematode infections. Infect Immun 71: 2430-2438. 